\section{Fatores associados à duração do aleitamento materno em crianças de famílias de baixa renda da região sul da cidade de Curitiba, Paraná, Brasil}

\author{
Factors associated with duration of breastfeeding \\ for children of low-income families from southern \\ Curitiba, Paraná State, Brazil
}

\footnotetext{
${ }^{1}$ Departamento de Estatística, Universidade Federal do Paraná, Curitiba, Brasil.

Correspondência S. R. Giolo Departamento de Estatística Universidade Federal do Paraná.

C. P. 19081, Curitiba, PR 81531-990, Brasil.

giolo@ufpr.br
}

\begin{abstract}
Various studies have focused on breastfeeding and the possible factors associated with early weaning. This study aimed to identify risk factors and protective factors for duration of breastfeeding in low-income families in southern Curitiba, Paraná State, Brazil. A cohort study from November 2006 to March 2007 included 118 mothers of children less than two years of age and enrolled in the TC Health Unit (USTC) located in the southern area of Curitiba. Statistical analyses using the Kaplan-Meier method and Cox regression model identified the following protective factors for breastfeeding: mother's awareness of the benefits of extended breastfeeding, exclusive feeding with mother's breast milk while in the maternity ward, and rooming-in. Factors contributing to early weaning were low birth weight, mother's work outside the home, and difficulties experienced by the mother in breastfeeding during the first days postpartum.
\end{abstract}

Breast Feeding; Weaning; Infant
Gerson Henrique Baptista ${ }^{1}$

Adriano Herbert H. K. Gonçalves de Andrade 1 Suely Ruiz Giolo 1

\section{Introdução}

Diversos estudos sobre amamentação 1,2,3,4,5,6, $7,8,9$, realizados no Brasil e em outros países desde meados da década de 1980, trouxeram contribuições relevantes para um melhor entendimento dos benefícios do aleitamento materno, especialmente do aleitamento materno exclusivo, tanto para a criança, quanto para a mulher. Os achados de muitos desses trabalhos produziram evidências científicas que levaram a reformulações das políticas nacionais e internacionais, as quais, em suas novas diretrizes, passaram a recomendar a amamentação exclusiva até o sexto mês 10,11,12,13,14,15. Atualmente, a Política Nacional de Aleitamento Materno tem como objetivos promover, proteger e apoiar a prática de aleitamento materno exclusivo até os seis meses e de complementação até os dois anos de idade ou mais 13 .

Os estudos nesse tema têm mostrado que vários são os benefícios do aleitamento materno, não só para a mãe e para a criança, como também para a família, a instituição e a sociedade. Um artigo recente que reúne uma seleção de estudos publicados nas duas últimas décadas traz uma revisão interessante sobre os benefícios da amamentação para a saúde da mãe e da criança 16. No caso da mãe, por exemplo, há menor sangramento pós-parto e, conseqüentemente, menor incidência de anemias e retardo na volta da menstruação; em virtude do maior intervalo interpartal, há menor prevalência de câncer de 
mama, ovário e endométrio, menos fraturas ósseas por osteoporose e maior rapidez na perda de peso pós-parto 4,10,16. Para a criança também são relatados vários benefícios, como menores índices de mortalidade, morbidade por diarréia, desnutrição, doenças respiratórias, otites, diabetes mellitus, alergias em geral, dermatite atópica, rinite alérgica e obesidade 1,2,10. Além disso, há indicações de que crianças amamentadas ao peito apresentam melhores índices de acuidade visual, desenvolvimento neuromotor, desenvolvimento cognitivo e quociente intelectual. Já para a família, a instituição e a sociedade, observamse, também, algumas vantagens, como economia com alimentação do recém-nascido e medicamentos; redução dos gastos institucionais com aquisição de fórmulas, frascos, bicos artificiais e medicamentos; redução da poluição ambiental (menos lixo inorgânico resultante do consumo de bicos artificiais e de mamadeiras).

Com a finalidade de conhecer a prática do aleitamento materno e identificar fatores associados à sua duração em crianças de famílias de baixa renda residentes na região sul da cidade de Curitiba, Paraná, Brasil, são apresentados, neste trabalho, os achados de um estudo de coorte realizado com 118 mães de crianças menores de dois anos de idade pertencentes ao Programa do Lactente da Unidade de Saúde TC (USTC). Os resultados deste estudo são de interesse da USTC, pois permitirão avaliar a duração do aleitamento materno das crianças que freqüentam a unidade, intensificando e promovendo ações de incentivo e apoio ao aleitamento. Na operacionalização dessas ações, serão priorizados os fatores que podem estar influenciando a duração do aleitamento materno.

\section{Material e métodos}

Para avaliar os fatores de risco ou proteção que influenciam a duração do aleitamento materno em crianças de famílias de baixa renda moradoras na região sul de Curitiba, foi adotado um questionário de acompanhamento das mães e de suas respectivas crianças composto de um total de 14 questões, sendo 13 relacionadas aos fatores de risco ou proteção de interesse (Tabela 1) e uma, ao desfecho (tempo ou duração da amamentação, em meses). O questionário foi elaborado com o auxílio da equipe de enfermagem da USTC, e as questões foram definidas conforme os objetivos principais do estudo: conhecer a prática do aleitamento materno e identificar fatores associados ao desmame precoce das crianças em estudo. Consultas a questionários utilizados em outras pesquisas que tiveram objetivos similares
7,8,9,17 foram realizadas a fim de auxiliar na definição das questões de interesse. Compuseram o questionário questões associadas à criança (sexo, peso ao nascimento, internações nos primeiros seis meses com separação da mãe, recebimento de leite exclusivamente materno na maternidade, alojamento com a mãe logo após o nascimento), à mãe (idade, nível educacional, experiência anterior com amamentação, número de filhos vivos, dificuldades para amamentar nos primeiros dias pós-parto, trabalho fora de casa, conceito do tempo ideal de amamentação) e à família (apoio emocional dos pais/avós e auxílio nas tarefas domésticas após o parto).

Participaram do estudo 118 mães de crianças menores de dois anos de idade, compreendendo em torno de $70 \%$ das mães com cadastro definitivo no Programa do Lactente da USTC entre novembro de 2006 e março de 2007. Não participaram somente aquelas com as quais não foi possível manter contato por terem mudado de endereço. As mães de crianças de até um ano de idade foram entrevistadas mensalmente na USTC, durante sua participação no Programa do Lactente, cujo objetivo é fazer um acompanhamento mensal da saúde do bebê. As demais mães, com crianças em geral maiores de um ano, que freqüentam a unidade com menor assiduidade, foram entrevistadas mensalmente em suas casas por agentes comunitárias de saúde, as quais foram orientadas e treinadas para o preenchimento adequado do questionário. A informação relativa à situação do aleitamento materno foi atualizada pelas agentes comunitárias de saúde todo final de mês. O registro da duração da amamentação das crianças que já não mais estavam sendo amamentadas à época do estudo foi feito com base nas informações fornecidas pelas mães, conforme suas memórias sobre o fato.

Quanto aos procedimentos de análise dos dados, foi inicialmente utilizado o método de Kaplan-Meier 18 a fim de se avaliarem quais dos fatores de risco ou proteção apresentados na Tabela 1 estariam associados à duração do aleitamento materno. De acordo com esse método 18 , a probabilidade de uma criança apresentar uma duração de aleitamento materno superior a $t$ meses é dada por:

$\mathrm{S}(t)=\prod_{j: t,<t}\left(1-\frac{d_{j}}{n_{j}}\right)$,

em que $t_{1}<t_{2} \ldots<t_{k}$ correspondem aos $k$ tempos, distintos e ordenados, das crianças que deixaram de ser amamentadas com leite materno; $d_{j}$ é o número de crianças que deixaram de ser amamentadas no tempo $t_{j}, j=1, \ldots, k ; n_{j}$ é o número de crianças em acompanhamento e em amamentação até o tempo imediatamente anterior a $t_{j}$. 
Tabela 1

Descrição dos fatores de risco ou proteção utilizados no estudo de aleitamento materno realizado na região sul de Curitiba, Paraná, Brasil, com suas respectivas freqüências absolutas e relativas.

\begin{tabular}{|c|c|c|c|}
\hline Código & Descrição do fator/Categorias & $\mathbf{n}$ & $\%$ \\
\hline \multirow[t]{3}{*}{$\mathrm{V} 1$} & Sexo da criança & & \\
\hline & 0 - Feminino & 58 & 49,15 \\
\hline & 1 - Masculino & 60 & 50,85 \\
\hline \multirow[t]{3}{*}{$\mathrm{V} 2$} & Experiência anterior da mãe com amamentação & & \\
\hline & 0 - Não & 55 & 46,61 \\
\hline & $1-\operatorname{Sim}$ & 63 & 53,39 \\
\hline \multirow[t]{3}{*}{ V3 } & Número de filhos vivos & & \\
\hline & 0 - Mais de 1 & 68 & 57,63 \\
\hline & $1-1$ & 50 & 42,37 \\
\hline \multirow[t]{3}{*}{ V4 } & Conceito materno sobre o tempo ideal de amamentação & & \\
\hline & 0 - Mais de 12 meses & 52 & 44,07 \\
\hline & $1-12$ meses ou menos & 66 & 55,93 \\
\hline \multirow[t]{3}{*}{ V5 } & Dificuldades da mãe para amamentar nos primeiros dias pós-parto & & \\
\hline & 0 - Não & 58 & 49,15 \\
\hline & $1-\operatorname{Sim}$ & 60 & 50,85 \\
\hline \multirow[t]{3}{*}{ V6 } & Recebimento de leite exclusivamente materno na maternidade & & \\
\hline & 0 - Não & 12 & 10,17 \\
\hline & $1-\operatorname{Sim}$ & 106 & 89,83 \\
\hline \multirow[t]{3}{*}{ V7 } & Peso da criança ao nascer & & \\
\hline & 0 - Mais de $2,5 \mathrm{~kg}$ & 103 & 87,29 \\
\hline & $1-2,5 \mathrm{~kg}$ ou menos & 15 & 12,71 \\
\hline \multirow[t]{3}{*}{ V8 } & Alojamento conjunto mãe/criança após o parto & & \\
\hline & $0-$ Não & 13 & 11,01 \\
\hline & $1-\operatorname{Sim}$ & 105 & 88,99 \\
\hline \multirow[t]{3}{*}{ V9 } & Apoio familiar (emocional e ajuda nas tarefas domésticas após o parto) & & \\
\hline & 0 - Não & 5 & 4,24 \\
\hline & $1-\operatorname{Sim}$ & 113 & 95,76 \\
\hline \multirow[t]{3}{*}{ V10 } & Idade da mãe & & \\
\hline & 0 - Mais de 25 anos & 57 & 48,31 \\
\hline & $1-25$ anos ou menos & 61 & 51,69 \\
\hline \multirow[t]{3}{*}{ V11 } & Internações da criança nos primeiros seis meses com separação da mãe & & \\
\hline & 0 - Não & 110 & 93,22 \\
\hline & $1-\operatorname{Sim}$ & 8 & 6,78 \\
\hline \multirow[t]{3}{*}{ V12 } & Nível educacional da mãe & & \\
\hline & 0 - Ensino Médio & 48 & 40,68 \\
\hline & 1 - Ensino Fundamental & 70 & 59,32 \\
\hline \multirow[t]{3}{*}{ V13 } & Mãe trabalha fora de casa & & \\
\hline & 0 - Não & 73 & 61,86 \\
\hline & $1-\operatorname{Sim}$ & 45 & 38,14 \\
\hline
\end{tabular}

Para testar se a duração de amamentação diferiu entre as categorias de um fator de risco ou proteção (por exemplo, entre os sexos masculino e feminino), foi utilizado o teste log rank proposto por Mantel 19 e também o teste de Wilcoxon 20.

Como o estimador de Kaplan-Meier 18 apresenta algumas limitações em estudos que envolvem diversos fatores de risco, foi também utilizado o modelo de Cox 21, que modela a função de risco dada por: $\lambda(t)=\lambda_{0}(t) \exp \left(\beta^{\prime} x\right)$

(2) em que $\lambda_{0}(t)$ é uma função do tempo não-especificada, denominada de função de risco de base, e $\beta$ é um vetor de parâmetros desconhecidos associados aos fatores de risco $\mathrm{x}$.

A suposição de que os riscos são proporcionais 22,23,24 é necessária para que o modelo (2) possa ser utilizado. A estimação dos parâmetros $\beta$ do modelo (2) é realizada por meio do método da máxima verossimilhança parcial 25 , sendo o 
exponencial de cada parâmetro interpretado como uma razão de riscos.

A partir do ajuste do modelo (2), pode-se avaliar a significância de um fator de risco na presença dos demais, bem como a significância de interações entre os fatores. Neste trabalho, a seleção dos fatores significativos foi feita com base em um método derivado da proposta de Collet 17,26, que é similar aos procedimentos stepwise 27 , mas que envolve uma participação mais ativa do estatístico e pesquisador em cada passo do processo de seleção. A suposição de riscos proporcionais foi avaliada por meio das curvas do logaritmo dos riscos acumulado 28 para cada fator no modelo final e, também, por meio dos resíduos de Schoenfeld 23,24.

Os resultados apresentados neste estudo foram obtidos com o auxílio do pacote estatístico R (The R Foundation for Statistical Computing, Viena, Áustria; http://www.r-project.org), gratuito e de domínio público.

\section{Resultados e discussão}

Na Tabela 1, são apresentados os fatores de risco ou proteção avaliados no presente estudo com as respectivas freqüências absolutas e relativas de mães ou crianças em cada uma das categorias desses fatores.

É possível observar que quase metade das mães não tinha qualquer experiência anterior com amamentação e metade encontrou dificuldades para amamentar nos primeiros dias pós-parto. Verifica-se, ainda, que praticamente a maioria das mães contou tanto com o apoio emocional dos pais e/ou avós, quanto com a ajuda de familiares nas tarefas domésticas após o nascimento da criança. Tem-se, também, pouco mais da metade $(57,63 \%)$ com mais de um filho vivo e $44 \%$ com consciência sobre os benefícios do aleitamento materno por tempo superior a 12 meses. Cerca de metade tinha idade inferior a 25 anos; $41 \%$ apresentaram nível educacional médio e as demais, nível fundamental; $38 \%$ trabalhavam fora.

Quanto às crianças, em torno da metade era do sexo feminino; aproximadamente $90 \%$ receberam leite exclusivamente materno na maternidade; $88 \%$ nasceram com peso superior a $2,5 \mathrm{~kg}$; $89 \%$ permaneceram alojadas na maternidade com a mãe e a maioria $(93,22 \%)$ não passou por internações, com separação da mãe, nos primeiros seis meses.

No que se refere à duração da amamentação das crianças, foi observado que $64(54,2 \%)$ estavam sendo amamentadas em março de 2007, data pré-estabelecida para o término do estudo e início das análises estatísticas. Os tempos de amamentação dessas 64 crianças são classificados na literatura como censuras 17 , tendo sido considerados como tal nas análises estatísticas.

Valendo-se do estimador de Kaplan-Meier 18 e dos testes $\log$ rank 19 e Wilcoxon ${ }^{20}$, avaliou-se individualmente cada fator de risco ou proteção (Tabela 1), a fim de que fossem obtidas indicações daqueles associados à duração da amamentação. A Figura 1 apresenta as curvas de KaplanMeier estimadas para os seguintes fatores: conceito materno sobre a duração (tempo) ideal de amamentação (V4) e dificuldades encontradas pela mãe para amamentar nos primeiros dias pós-parto (V5). Inspeção visual das curvas mostradas nessa figura revela indícios de que estas diferem entre si. Embora não apresentadas, curvas de sobrevivência foram também obtidas para todos os demais fatores.

Na Tabela 2, que mostra os resultados dos testes log rank e Wilcoxon utilizados para testar as diferenças das curvas para esses e os demais fatores mostrados na Tabela 1, pode-se observar os menores valores $\mathrm{p}$ associados aos fatores V4, V5, V6 (recebimento de leite exclusivamente materno na maternidade), V7 (peso da criança ao nascimento), V8 (alojamento conjunto mãe/criança na maternidade) e V13 (trabalho da mãe fora de casa). Estes são, dentre os avaliados, os fatores que possivelmente estão associados ao tempo de aleitamento materno das crianças neste estudo.

A fim de se avaliarem conjuntamente os fatores de risco e interações dois a dois entre eles, ajustou-se o modelo de Cox 21 . Os resultados foram concordantes com os obtidos quando da utilização do método de Kaplan-Meier e forneceram evidências de que os mesmos seis fatores de risco ou proteção encontrados anteriormente (V4, V5, V6, V7, V8 e V13) estariam associados ao tempo de amamentação. Nenhuma interação de segunda ordem entre os fatores foi significativa. Na Tabela 3, são apresentadas as estimativas dos parâmetros do modelo de Cox relativas aos seis fatores de risco significativos, bem como as correspondentes razões de risco e seus respectivos intervalos de 95\% de confiança.

A suposição de riscos proporcionais associada ao modelo de Cox foi considerada adequada. Para tanto, levaram-se em conta não só os gráficos do logaritmo da função do risco acumulado $\log \left(\Lambda_{0}(t)\right)$ versus o tempo, mostrados na Figura 2 para cada fator de risco incluído no modelo final, que não apresentaram cruzamentos marcantes entre as curvas, como também a análise dos resíduos padronizados de Schoenfeld (não apresentados).

As estimativas dos efeitos dos fatores associados ao tempo de aleitamento materno (Tabe- 
Figura 1

Curvas de sobrevivência $S(t)$ estimadas por Kaplan-Meier para os fatores: conceito materno sobre o tempo de amamentação (V4) e dificuldades para amamentar nos primeiros dias pós-parto (V5)
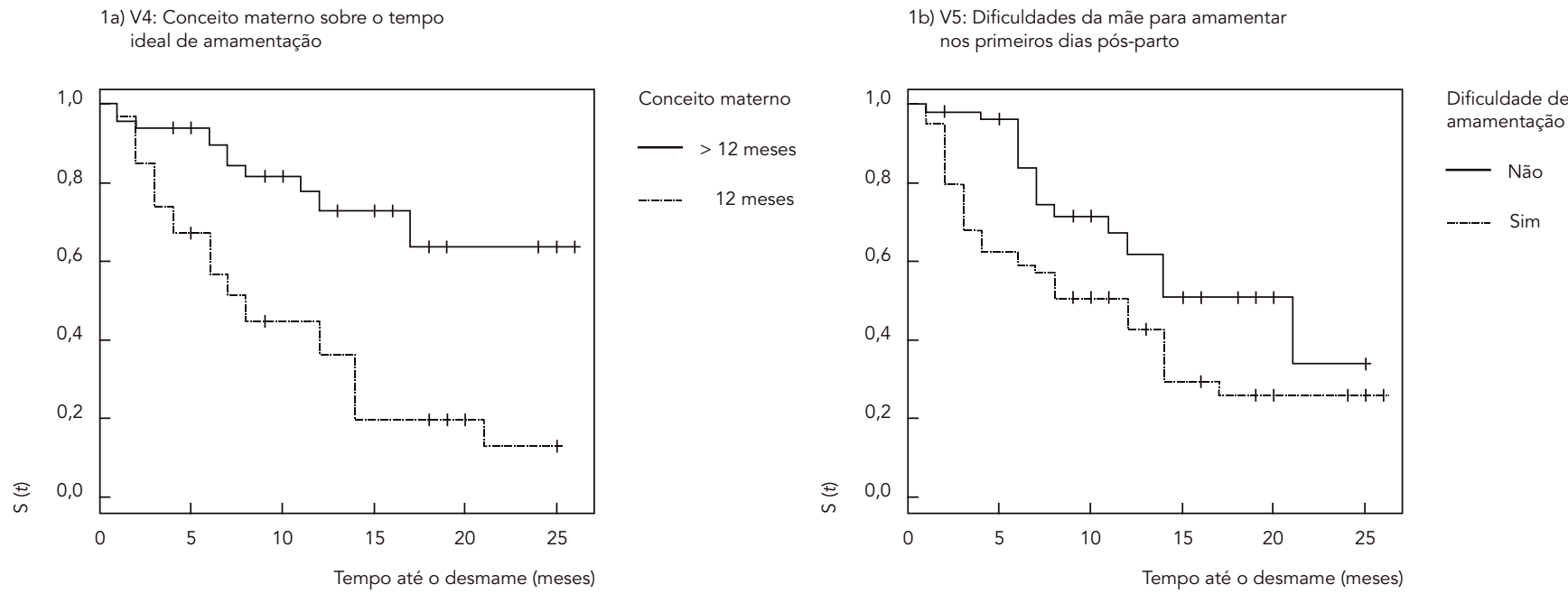

Tabela 2

Resultados dos testes log rank e Wilcoxon, utilizados para testar as curvas de sobrevivência obtidas para cada fator, por meio do estimador de Kaplan-Meier.

\begin{tabular}{lcc}
\hline Fator de risco ou proteção avaliado & \multicolumn{2}{c}{ Testes (valor de p) } \\
& Log rank & Wilcoxon \\
\hline V1: Sexo da criança & $0,770(0,380)$ & $0,773(0,379)$ \\
V2: Experiência anterior da mãe com amamentação & $0,008(0,929)$ & $0,005(0,946)$ \\
V3: Número de filhos vivos & $0,019(0,891)$ & $0,040(0,842)$ \\
V4: Conceito materno sobre o tempo ideal de amamentação & $19,100(<0,001)$ & $18,100(<0,001)$ \\
V5: Dificuldade para amamentar nos primeiros dias pós-parto & $7,310(0,007)$ & $9,710(0,002)$ \\
V6: Recebeu leite exclusivamente materno na maternidade & $1,910(0,166)$ & $2,880(0,090)$ \\
V7: Peso da criança ao nascimento & $11,200(<0,001)$ & $12,400(<0,001)$ \\
V8: Alojamento conjunto mãe/criança & $11,800(<0,001)$ & $10,800(0,001)$ \\
V9: Apoio familiar & $0,876(0,349)$ & $1,190(0,275)$ \\
V10: Idade da mãe & $0,262(0,609)$ & $0,033(0,856)$ \\
V11: Internações nos primeiros seis meses com separação & $0,226(0,635)$ & $1,310(0,252)$ \\
V12: Nível educacional da mãe & $1,290(0,257)$ & $0,722(0,395)$ \\
V13: Mãe trabalha fora de casa & $5,140(0,023)$ & $4,530(0,033)$ \\
\hline
\end{tabular}

la 3) mostram que o risco de desmame precoce de crianças com peso ao nascimento inferior ou igual a 2,5kg é, aproximadamente, quatro vezes maior do que o das crianças com peso ao nascimento superior a $2,5 \mathrm{~kg}$. Com $95 \%$ de confiança, esse risco pode variar entre 1,78 e 9,35. O risco de desmame precoce é também maior entre as crianças (a) cujas mães acreditam que o tempo ideal de amamentação é inferior ou igual a 12 meses, (b) que não receberam leite exclusivamente materno na maternidade, (c) que não tiveram alojamento conjunto na maternidade e (d) cujas mães trabalham fora de casa. Foi, também, observada uma tendência ( $p=0,085$ : modelo de 
Estimativas resultantes do ajuste do modelo de Cox aos dados de aleitamento materno, razões de risco (RR) e respectivos intervalos de $95 \%$ de confiança (IC95\%).

\begin{tabular}{lccccc}
\hline Covariável & Estimativa & Erro padrão & Valor de p & RR & IC95\% (RR) \\
\hline V4 & 1,529 & 0,371 & $<0,0001$ & 4,61 & $(2,23 ; 9,54)$ \\
V5 & 0,526 & 0,306 & 0,085 & 1,69 & $(0,93 ; 3,08)$ \\
V6 & $-1,076$ & 0,465 & 0,021 & 0,34 & $(0,14 ; 0,85)$ \\
V7 & 1,405 & 0,424 & 0,001 & 4,08 & $(1,78 ; 9,35)$ \\
V8 & $-0,983$ & 0,426 & 0,021 & 0,37 & $(0,16 ; 0,86)$ \\
V13 & 0,581 & 0,285 & 0,042 & 1,79 & $(1,02 ; 3,13)$ \\
\hline
\end{tabular}

Figura 2

Gráficos de $\log \left(\Lambda_{0}(t)\right)$ versus tempo (em meses) para os fatores que apresentaram significância estatística no modelo de Cox (V4, V5, V6, V7, V8 e V13), sendo $\Lambda_{0}(t)$ o risco de base acumulado.

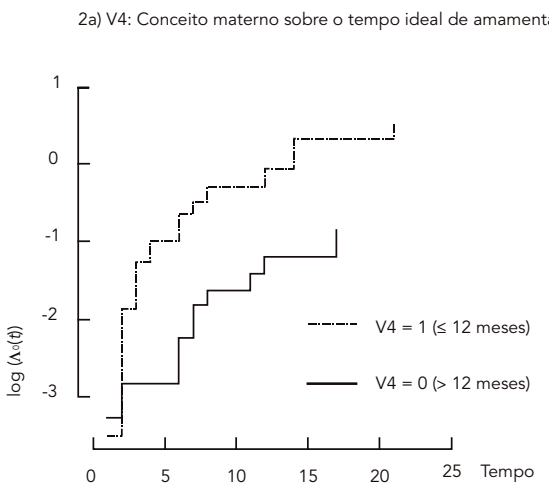

2d) V7: Peso da criança ao nascer

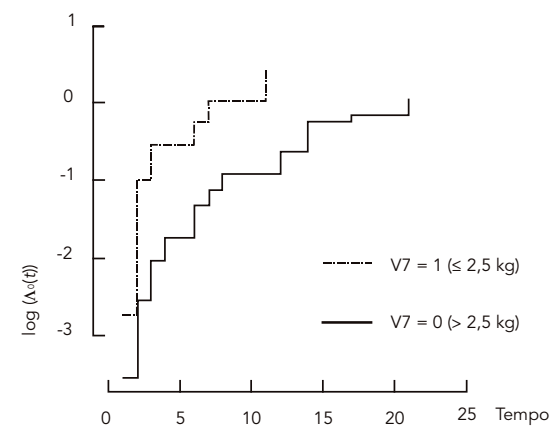

2b) V5: Dificuldades da mãe para amamentar nos primeiros dias pós-parto

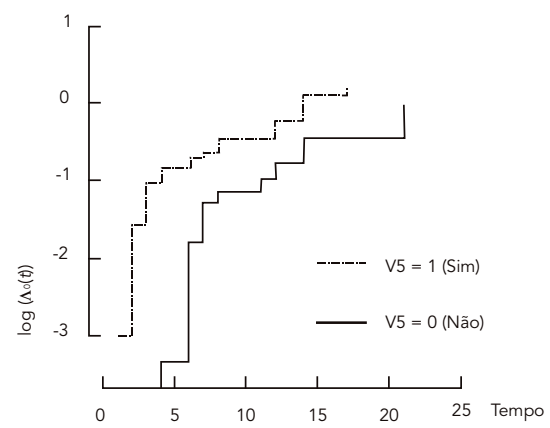

2e) V8: Alojamento conjunto mãe/criança após o parto

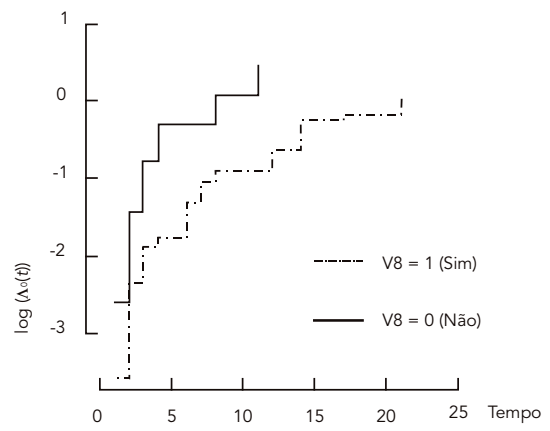

2c) V6: Recebimento de leite exclusivamente materno na maternidade

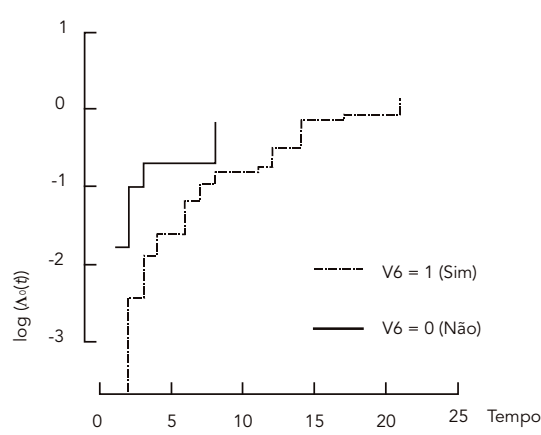

2f) V13: Mãe trabalha fora de casa

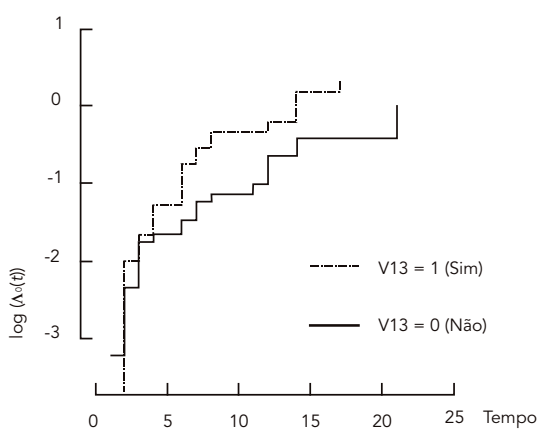

Cox e p < 0,01: Kaplan-Meier) de tempo menor de amamentação para mulheres que apresentaram dificuldades para amamentar nos primeiros dias pós-parto.
Em síntese, os resultados do estudo analisado neste trabalho apontaram como fatores protetores do aleitamento materno: o conceito materno sobre o tempo ideal de amamentação, 
o recebimento de leite exclusivamente materno na maternidade e o alojamento conjunto, mãe e criança, na maternidade. Por outro lado, os resultados apontaram os seguintes fatores como os que contribuem para o desmame precoce: o baixo peso da criança ao nascimento, o fato de a mãe trabalhar fora de casa e as dificuldades encontradas pela mãe para amamentar nos primeiros dias pós-parto.

Neste trabalho, foi também observado que alguns fatores não apresentaram grande variabilidade (por exemplo, apoio familiar). Isso evidencia que as mulheres estudadas pertencem a famílias que apresentam um comportamento muito semelhante no que diz respeito ao apoio dado à mãe. Em outras regiões ou cidades, esse apoio familiar pode ser menos evidente, podendo vir a influenciar a duração do aleitamento materno. Notou-se, ainda, um percentual elevado de censuras (54\%); por isso, sugere-se que a equipe da USTC continue a acompanhar as mães por um período de tempo maior a fim de se reduzir este percentual e, então, obter uma avaliação mais precisa da duração do aleitamento materno na amostra de mães sendo analisada.

Tendo em vista as diferenças existentes entre as populações avaliadas em diferentes estudos, é difícil fazeruma comparação direta dos resultados deste trabalho com os de outros. Ainda assim, foi possível notar, entre eles, alguns fatores comuns associados à duração do aleitamento materno: o peso das crianças ao nascimento 7,29 , a amamentação nas primeiras horas pós-parto 17,30 , o conceito materno sobre o tempo ideal de amamentação e as dificuldades para amamentar nos primeiros dias pós-parto ${ }^{17}$.

Em face dos resultados obtidos no estudo analisado neste trabalho, a USTC decidiu manter a observação direta e a orientação às mães acompanhadas no Programa do Lactente desenvolvido na unidade. Os membros da equipe da USTC decidiram, também, priorizar o tema, submetendo toda a equipe técnica e os demais funcionários a treinamentos ministrados pelo PROAMA (Programa de Aleitamento Materno) de Curitiba e pelo Amamenta Brasil, do Governo Federal. Como produto principal dessas ações de treinamento, implantou-se um Banco de Leite na USTC e desenvolveu-se um trabalho de incentivo à doação de leite humano ao Banco de Leite da cidade. Os resultados do estudo foram, portanto, importantes para o direcionamento das ações da USTC, servindo de referência para a equipe centralizar a sua atenção nos fatores de risco com maior associação ao desmame precoce. Cita-se como exemplo o conceito materno sobre o tempo ideal de amamentação (V4). Neste caso, a equipe procurou priorizar e dar maior ênfase à conscientização das mães quanto à importância e aos benefícios do aleitamento materno prolongado.

\section{Resumo}

O aleitamento materno e os possíveis fatores associados ao desmame precoce têm sido alvos de diversos estudos. Este trabalho teve como objetivo identificar, em famílias de baixa renda da região sul de Curitiba, Paraná, Brasil, fatores de risco ou de proteção associados à duração do aleitamento materno. Um estudo de coorte foi conduzido entre novembro de 2006 e março de 2007 com 118 mães de crianças menores de dois anos de idade com vínculo na Unidade de Saúde TC (USTC), localizada na região sul de Curitiba. Análises estatísticas realizadas com o auxílio do método de Kaplan-Meier e do modelo de regressão de Cox apontaram como fatores protetores do aleitamento materno: a consciência da mãe sobre os benefícios da amamentação por tempo estendido, a amamentação da criança com leite exclusivamente materno na maternidade e o alojamento conjunto da mãe e criança na maternidade. Por outro lado, o baixo peso da criança ao nascimento, o trabalho da mãe fora de casa e as dificuldades encontradas pela mãe para amamentar nos primeiros dias pós-parto foram fatores apontados como contribuidores do desmame precoce.

Aleitamento Materno; Desmame; Lactente 


\section{Colaboradores}

G. H. Baptista participou da elaboração e aplicação do questionário e da coleta dos dados. G. H. Baptista, A. H. H. K. G. Andrade e S. R. Giolo analisaram os dados, interpretaram os resultados e participaram da elaboração do artigo.

\section{Agradecimentos}

À equipe de enfermagem da Unidade de Saúde TC de Curitiba, pela participação na elaboração e aplicação do questionário. Às agentes comunitárias de saúde, pela colaboração na coleta dos dados.

\section{Referências}

1. Victoria CG, Smith PG, Vaughan JP, Nobre LC, Lombardi C, Teixeira AM, et al. Evidence for protection by breast-feeding against infant deaths from infectious diseases in Brazil. Lancet 1987; 2:319-22.

2. Popkin BM, Adair L, Akin JS, Black R, Briscoe J, Flieger W. Breast-feeding and diarrheal morbidity. Pediatrics, 1990; 86:874-82.

3. Labbok MH, Wardlaw T, Blanc A, Clark D, Terreri $\mathrm{N}$. Trends in exclusive breastfeeding: findings from the 1990s. J Hum Lact 2006; 22:272-6.

4. Kramer MS, Kakuma R. The optimal duration of exclusive breastfeeding: a systematic review. Adv Exp Med Biol 2004; 554:63-77.

5. Bahl R, Frost C, Kirkwood BR, Edmond K, Martines J, Bhandari N, et al. Infant feeding patterns and risks of death and hospitalization in the first half of infancy: multicentre cohort study. Bull World Health Organ 2005; 83:418-26.

6. Leão MM, Coitinho DC, Recine E, Costa LAL, Lacerda AJ. O perfil do aleitamento materno no Brasil. In: Instituto Brasileiro de Geografia e Estatística/Instituto Nacional de Alimentação e Nutrição/Fundo das Nações Unidas para a Infância, organizadores. Perfil estatístico de crianças e mães no Brasil: aspectos de saúde e nutrição de crianças no Brasil, 1989. Rio de Janeiro: Instituto Brasileiro de Geografia e Estatística; 1992. p. 97-110.
7. Gigante DP, Victora CG, Barros FC. Nutrição materna e duração da amamentação em uma coorte de nascimento de Pelotas, RS. Rev Saúde Pública 2000; 34:259-65.

8. Lima TM, Osório MM. Perfil e fatores associados ao aleitamento materno em crianças menores de 25 meses da Região Nordeste do Brasil. Rev Bras Saúde Matern Infant 2003; 3:305-14.

9. Silveira FJS, Lamounier JA. Fatores associados à duração do aleitamento materno em três municípios na região do Alto Jequitinhonha, Minas Gerais, Brasil. Cad Saúde Pública 2006; 22:69-77.

10. Organização Mundial da Saúde. Aleitamento materno: proteção, promoção e apoio. O papel especial dos serviços materno-infantis. Femina 1992; 2:969-4.

11. Organização Mundial da Saúde. Estratégia global para a alimentação de lactentes e crianças de primeira infância. http://www.ibfan.org.br/documentos (acessado em 25/Ago/2008).

12. Organização Mundial da Saúde. Evidências científicas dos dez passos para o sucesso no aleitamento materno. Brasília: Organização Pan-Americana da Saúde/Organização Mundial da Saúde; 2001.

13. Secretaria de Políticas de Saúde, Ministério da Saúde. Guia alimentar para crianças menores de 2 anos. Brasília: Ministério da Saúde; 2002. 
14. UNICEF Innocenti Research Centre. Celebrating the Innocenti Declaration on the Protection, Promotion and Support of Breastfeeding past achievements, present challenges and the way forward for infant and young child feeding. Florence: UNICEF Innocent Research Centre; 2005.

15. Corintio Mariani Neto, editor. Federação Brasileira das Associações de Ginecologia e Obstetrícia. Aleitamento materno: manual de orientação. São Paulo: Ponto; 2006.

16. Toma TS, Rea MF. Benefícios da amamentação para a saúde da mulher e da criança: um ensaio sobre as evidências. Cad Saúde Pública 2008; 24 Suppl 2:S235-46.

17. Colosimo EA, Giolo SR. Análise de sobrevivência aplicada. São Paulo: Edgard Blücher; 2006.

18. Kaplan EL, Meier P. Nonparametric estimation from incomplete observations. J Am Stat Assoc 1958; 53:457-81

19. Mantel N. Evaluation of survival data and two new rank order statistics arising in its consideration. Cancer Chemother Rep 1966; 6:163-8.

20. Gehan EA. A generalized Wilcoxon test for comparing arbitrarily singly-censored samples. Biometrika 1965; 52:203-21.

21. Cox DR. Regression models and life-tables. Journal of the Royal Statistical Society, Series B 1972; 34:187-202
22. Grambsch PM, Therneau TM. Proportional hazards tests and diagnostics based on weighted residuals. Biometrika 1994; 81:515-26.

23. Schoenfeld DA. Chi-squared goodness of fit tests for the proportional hazards regression model. Biometrika 1980; 67:145-9.

24. Schoenfeld D. Partial residuals for the propor tional hazards regression model. Biometrika 1982; 69:239-41.

25. Cox DR. Partial likelihood. Biometrika 1975; 62:269-76.

26. Collet D. Modelling survival data in medical research. New York: Chapman \& Hall; 1994.

27. Draper NR, Smith H. Applied regression analysis. New York: John Wiley \& Sons; 1998

28. Breslow N. Contribuição à discussão do artigo de D.R. Cox. Journal of the Royal Statistical Society Series B 1972; 34:216-7.

29. Victora CG, Huttly SRA, Barros FC, Vaughan JP. Cesarean section and duration of breastfeeding among Brazilians. Arch Dis Child 1990; 65:632-4.

30. Martines JC, Ashworth A, Kirkwood B. Breast-feeding among the urban poor in southern Brazil: reasons for termination in the first 6 months of life. Bull World Health Organ 1989; 67:151-61.

Recebido em 27/Fev/2008

Versão final reapresentada em 28/Ago/2008

Aprovado em 29/Set/2008 